\title{
A randomised controlled trial comparing oxytocin and oxytocin + ergometrine for prevention of postpartum haemorrhage at caesarean section
}

\author{
S Koen, BSc, MB BCh; L C Snyman, BMedSci, MB ChB, MMed (O\&G), FCOG (SA), MPraxMed; \\ R C Pattinson, BSc, MB BCh, FRCOG, MD, FCOG (SA), MMed (O\&G), MRCOG; J A Makin, MB BCh, MSc (Clinical Epidemiology)
}

Department of Obstetrics and Gynaecology, Faculty of Health Sciences, University of Pretoria, South Africa

Corresponding author: S Koen (sandy.koen811@gmail.com)

\begin{abstract}
Background. Globally 166000 women die annually as a result of obstetric haemorrhage. More than $50 \%$ of these deaths occur in subSaharan Africa. Uterine atony is the commonest cause of severe postpartum haemorrhage (PPH). Bleeding at or after caesarean section (CS) is responsible for $>30 \%$ of maternal deaths due to obstetric haemorrhage in South Africa (SA).

Objective. To compare oxytocin alone with oxytocin + ergometrine in terms of primary prophylaxis for PPH at the time of CS.

Methods. This was a double-blind randomised controlled interventional study comparing oxytocin with oxytocin + ergometrine administered during CS. Patients were randomised to receive oxytocin alone intravenously as a bolus or oxytocin + ergometrine intramuscularly, with the placebo being an injection of sterile water. The study population consisted of women undergoing CS at Kalafong Provincial Tertiary Hospital in Atteridgeville, Gauteng, SA.

Results. Five hundred and forty women were randomised and data for 416 women, of whom 214 received oxytocin and 202 oxytocin + ergometrine, were available for analysis. In the oxytocin group 19 women (8.9\%) required blood transfusion, compared with seven (3.5\%) in the oxytocin + ergometrine group ( $p=0.01$; relative risk $=2.78 ; 95 \%$ confidence interval $1.21-6.4$ ). There were no statistically significant differences in the mean estimated visual and mean calculated blood loss.

Conclusions. The overall need for blood transfusion was significantly reduced by about two-thirds in women receiving the oxytocin + ergometrine combination. Consideration should be given to using oxytocin + ergometrine for prophylaxis of PPH at CS.

S Afr Med J 2016;106(4):399-402. DOI:10.7196/SAMJ.2016.v106i4.10133
\end{abstract}

Globally 166000 women die annually as a result of obstetric haemorrhage. More than $50 \%$ of these deaths occur in sub-Saharan Africa. Uterine atony is the commonest cause of severe postpartum haemorrhage (PPH) ${ }^{[1]}$ The term 'significant obstetric haemorrhage', defining a loss of $>1500 \mathrm{~mL}$, can also be used to describe PPH. ${ }^{[2]}$

The preliminary data of the 2011 - 2013 Saving Mothers triennial report $^{[3]}$ revealed that obstetric haemorrhage accounted for 684 maternal deaths, making it the second most common cause of maternal death in South Africa (SA). One of the major concerns was bleeding after caesarean section (CS). The following major causes have been identified among the cohort of the 2011 - 2013 deaths ascribed to haemorrhage: ${ }^{[3]}(i)$ inadequate utilisation of uterotonic agents; (ii) poor recognition of the severity of the blood loss causing hypovolaemia; (iii) inadequate surgical skill; and (iv) delays in relaparotomy and/or referral in case of post-CS bleeding.

Oxytocin (Syntocinon; Sandoz) can be administered in several different ways during CS to prevent PPH. At Kalafong Provincial Tertiary Hospital (KPTH), Pretoria, SA, oxytocin $2.5 \mathrm{IU}$ is given as an intravenous bolus followed by $7.5 \mathrm{IU}$ in the remaining Vaculiter to be administered during the CS. According to the Green Top Guidelines No. 52 of the Royal College of Obstetrics and Gynaecology, ${ }^{[4]}$ the required initial bolus dose is $5 \mathrm{IU}$ slowly as an intravenous infusion (IVI). There has also been a recent publication that referred to the 'rule of threes' as a means of administering oxytocin: 3 IU IVI as a slow bolus every 3 minutes depending on the contraction of the uterus. ${ }^{[5]}$ The following guidelines were published in the SAMJ in April 2015 for primary prophylaxis of PPH at CS:[6] (i) oxytocin 2.5 IU IVI as a slow bolus (over 30 seconds); (ii) oxytocin 7.5 IU in the remaining IVI fluid running in; and (iii) oxytocin infusion
$20 \mathrm{IU} / 1000 \mathrm{~mL}$ at $125 \mathrm{~mL} / \mathrm{h}$ for 8 hours $(125 \mathrm{~mL} / \mathrm{h}$ is equivalent to 42 drops per minute from a ' 20 dropper' administration set).

One of the key recommendations from the Saving Mothers report $2008-2010^{[7]}$ is to use the combination of oxytocin + ergometrine (Syntometrine; Sandoz) as the second-line treatment for uterine atony in preference to misoprostol, unless contraindicated. In addition, this combination is to be considered at CS as primary prophylaxis for $\mathrm{PPH}{ }^{[7]}$

Oxytocin + ergometrine has mainly been reserved as a secondline agent in the management of PPH at the time of CS. In view of the unacceptable number of maternal deaths due to obstetric haemorrhage following CS, it is important to investigate the use of alternative measures to prevent PPH at CS.

\section{Objective}

To compare the efficacy of oxytocin + ergometrine with that of oxytocin in the primary prevention of PPH at CS.

\section{Methods}

This was a double-blind placebo-controlled randomised study done at KPTH. All pregnant women aged $\geq 18$ years undergoing elective or emergency lower-segment CS, who were able to provide informed consent and did not have any of the exclusion criteria listed in Table 1, were eligible for recruitment to the study.

Pre- and postoperative measurement of the haematocrit and intraoperative estimated visual blood loss were used as indicators of blood loss. A questionnaire was used postoperatively to assess the side-effects experienced by patients recruited to the study. The need for blood transfusion and additional medication was also recorded. 
Patients were randomised to receive either $2.5 \mathrm{U}$ oxytocin alone intravenously as a bolus and placebo intramuscularly, or $5 \mathrm{U}$ oxytocin $+0.5 \mathrm{mg}$ ergometrine (1 ampoule of Syntometrine) intramuscularly and placebo intravenously, after delivery of the neonate. In both groups an additional $10 \mathrm{U}$ of oxytocin were added to the Vaculiter. The anaesthetists involved in the respective cases administered all drugs. Recruitment took place from 2 January 2014 until I June 2014, only when the research co-ordinator was on site.

Randomisation was by means of sealed nontransparent envelopes. Each had a label inside with a letter A (oxytocin group) or B (oxytocin + ergometrine), which corresponded to a pair of prepacked colour-coded ampoules that were used for the two different groups. Each pair of ampoules consisted of one ampoule with an active ingredient (oxytocin 2.5 IU alone or oxytocin $5 \mathrm{IU}+$ ergometrine 0.5 $\mathrm{mg}$ ), as well as a placebo ampoule containing sterile water for injection. The glass ampoules were covered in red and blue coloured tape to disguise the ingredients. The blue ampoules were administered intravenously and the red ampoules intramuscularly. Following randomisation into one of the two groups, the appropriate prepacked ampoules were handed to the anaesthetist for administration. The anaesthetists, obstetricians and study participants were all blinded as to what active ingredients were administered.

Table 1. Exclusion criteria
Women not willing or not able to provide
consent
Women who have had a classic CS
Women $<18$ years of age
Women with any of the following
conditions (ergometrine contraindicated in
patients with these conditions):
Pre-eclampsia
Eclampsia
Uncontrolled hypertension
(hypertension defined as systolic blood
pressure $>140$ mmHg and diastolic
blood pressure $>90$ mmHg)
Any cardiac lesion
Impaired liver function
Impaired kidney function
Hypersensitivity to any of the active
ingredients of the preparations that
will be used (oxytocin or oxytocin +
ergometrine)
Occlusive vascular disease
Autoimmune vasculitis

In the event of $\mathrm{PPH}$, treatment was per the standard protocol and guidelines. The active ingredient used to prevent PPH did not influence its treatment.

The sample size was calculated making use of a control value of severe $\mathrm{PPH}$ of $22 \%,{ }^{[3]}$ an absolute difference between control and intervention of $10 \%$ reduction in bleeding at CS suggesting that the intervention was effective. The resulting sample size was 221 women in each group.

Preoperative and postoperative (6 - 24 hours) haematocrit or haemoglobin values were measured. In some cases arterial blood gas haemoglobin levels were used. In addition, visual estimated blood loss was documented. Calculation of blood loss was done using calculated pregnancy preoperative blood volume $(0.75 \times[$ height inches $\times 50\}+\{$ weight pounds $\times 25\}) \times$ percentage of blood volume lost ([pre-delivery haematocrits - post-delivery haematocrits]/pre-delivery haematocrits), as described by Stratford et al. ${ }^{[8]}$

The need for additional uterotonics and the number of units of blood transfused were documented. The anaesthetist and obstetrician managing the individual patients made the decision for blood transfusion based on clinical assessment at the time of CS.
Data were entered into an Excel database for checking and editing. This was then imported into SPSS version 21 for analysis. Descriptive statistics were used to describe the population as a whole. Comparisons between patients receiving the intervention and the control group were made using independent-sample $t$-tests in the case of continuous data and $\chi^{2}$ tests in the case of categorical data.

The study was approved by the Research Ethics Committee, Faculty of Health Sciences, University of Pretoria (391/2013), and was registered on ClinicalTrials.gov (NCT02046499).

\section{Results}

Five hundred and forty women were randomised to the study (Fig. 1). Data on 124 patients who were randomised were excluded from analysis. Of these, 80 delivered vaginally and another 44 were excluded because of incomplete data or protocol violations, resulting in data on 416 women that were analysed. Two hundred and fourteen women were randomised to receive oxytocin alone and 202 received oxytocin + ergometrine.

The two groups of patients were analysed in terms of age, parity, gravidity and gestational ages in weeks. The results are shown in Table 2.
Table 2. Demographic data

\begin{tabular}{llll}
\hline & $\begin{array}{l}\text { O group } \\
(\boldsymbol{n}=\mathbf{2 1 4}), \text { mean }(\mathrm{SD})\end{array}$ & $\begin{array}{l}\text { O+E group } \\
(\boldsymbol{n}=\mathbf{2 0 2}) \text {, mean }(\mathrm{SD})\end{array}$ & $\boldsymbol{p}$-value \\
\hline Age (years) & $28.6(6.0)$ & $28.9(5.4)$ & 0.59 \\
Parity $(n)$ & $1.2(1.1)$ & $1.1(0.9)$ & 0.21 \\
Gravidity $(n)$ & $2.4(1.3)$ & $2.4(1.1)$ & 0.44 \\
Gestational age & $38.4(2.2)$ & $38.5(1.9)$ & 0.33 \\
(weeks) & & & \\
Height $(\mathrm{cm})$ & $159.4(15.2)$ & $160.2(11.3)$ & 0.57 \\
Weight $(\mathrm{kg})$ & $75.6(16.2)$ & $76.6(17.2)$ & 0.53 \\
MUAC $(\mathrm{cm})$ & $29.6(3.8)$ & $30.1(4.6)$ & 0.26 \\
O = oxytocin; O+E = oxytocin + ergometrine; SD = standard deviation; MUAC = mid-upper arm circumference.
\end{tabular}

Table 3. Indications for CS

\begin{tabular}{llll}
\hline Indication for CS & $\begin{array}{l}\text { O group } \\
(\boldsymbol{N = 2 1 4 )}, \boldsymbol{n}(\mathbf{\%})\end{array}$ & $\begin{array}{l}\text { O+E group } \\
(\boldsymbol{N = 2 0 2}), \boldsymbol{n}(\%)\end{array}$ & $\begin{array}{l}\text { Total } \\
(\boldsymbol{N}=\mathbf{4 1 6}), \boldsymbol{n}(\mathbf{\%})\end{array}$ \\
\hline Previous CS & $91(42.5)$ & $97(48.0)$ & $188(45.2)$ \\
Fetal distress & $49(22.9)$ & $38(18.8)$ & $87(20.9)$ \\
Cephalopelvic disproportion & $19(8.9)$ & $22(10.9)$ & $41(9.9)$ \\
Poor progress & $17(7.9)$ & $13(6.4)$ & $30(7.2)$ \\
Failed induction of labour & $6(2.8)$ & $8(4.0)$ & $14(3.4)$ \\
Breech & $9(4.2)$ & $5(2.5)$ & $14(3.4)$ \\
Twins & $5(2.3)$ & $6(3.0)$ & $11(2.6)$ \\
Other & $13(8.4)$ & $11(6.4)$ & $24(7.4)$ \\
O = oxytocin; O+E = oxytocin + ergometrine. & &
\end{tabular}


The most common indication for performing CS in the study population was a previous CS ( $n=188,45.2 \%)$. The indications for CS are listed in Table 3.

Women who underwent emergency CS were compared with those who had elective CS, and these results are shown in Table 4. Two hundred and sixty-seven women (64.2\%) underwent emergency CS and 149 (35.8\%) had elective CS.

Three hundred and ninety-three patients (94.5\%) were administered a spinal anaesthetic and $23(5.5 \%)$ a general anaesthetic. There were no statistically significant differences between the two groups $(p=0.721)$. Spinal anaesthesia was administered to 203 patients $(94.9 \%)$ in the oxytocin group and to $190(94.1 \%)$ in the oxytocin + ergometrine group.

The mean estimated visual blood loss in the oxytocin group was $607 \mathrm{~mL}$ and that in the oxytocin + ergometrine group $588 \mathrm{~mL}$ $(p=0.437)$.

Women who received intraoperative or immediate postoperative blood transfusions were excluded from the postoperative calculated blood loss analysis. Data on 371 of the 417 patients analysed were used for calculated blood loss. The preoperative values were not statistically different between the two groups $(p=0.707)$ and the postoperative haematocrit values also did not differ $(p=0.283)$. The oxytocin group had a mean postoperative haematocrit of 0.324 , while the mean postoperative haematocrit in the oxytocin + ergometrine group was 0.329 . The mean calculated blood loss in the oxytocin group was $610 \mathrm{~mL}$ (95\% confidence interval (CI) 0.321 $0.335)$ and that in the oxytocin + ergometrine group $590 \mathrm{~mL}$ ( $95 \%$ CI 0.326 - 0.338) $(p=0.445$ ).

The need for blood transfusion was calculated using $\chi^{2}$ tests. Nineteen women $(8.9 \%)$ in the oxytocin group required a blood transfusion as opposed to seven $(3.5 \%)$ in the oxytocin + ergometrine group $(p=0.01$; relative risk (RR) 2.78; 95\% CI 1.21 - 6.4)

The need for additional uterotonics was also recorded. In total, these were required by 36 patients (8.7\%), 16 (7.5\%) in the oxytocin group and $20(9.9 \%)$ in the oxytocin + ergometrine group. This difference was not significant $(p=0.379)$.
Two hundred and four women in the oxytocin group (95.3\%) experienced no bothersome side-effects, compared with 194 $(96.0 \%)$ in the oxytocin + ergometrine group $(p=0.81)$.

\section{Discussion}

Data from the latest Saving Mothers report ${ }^{[3]}$ suggest that maternal morbidity and mortality secondary to obstetric haemorrhage at CS is on an upward trend. It is useful to note that if the risk of death from vaginal delivery is 1 , the relative risk for all CSs is 4.9. The relative risk for elective CS is 2.3 compared with 12.0 when the caesarean is performed because of immediate threat to the life of the mother or fetus. ${ }^{[9]}$ For this reason, administration of uterotonic drugs during CS has become of paramount importance in prophylaxis as a strategy for improving maternal morbidity and mortality. ${ }^{[10]}$

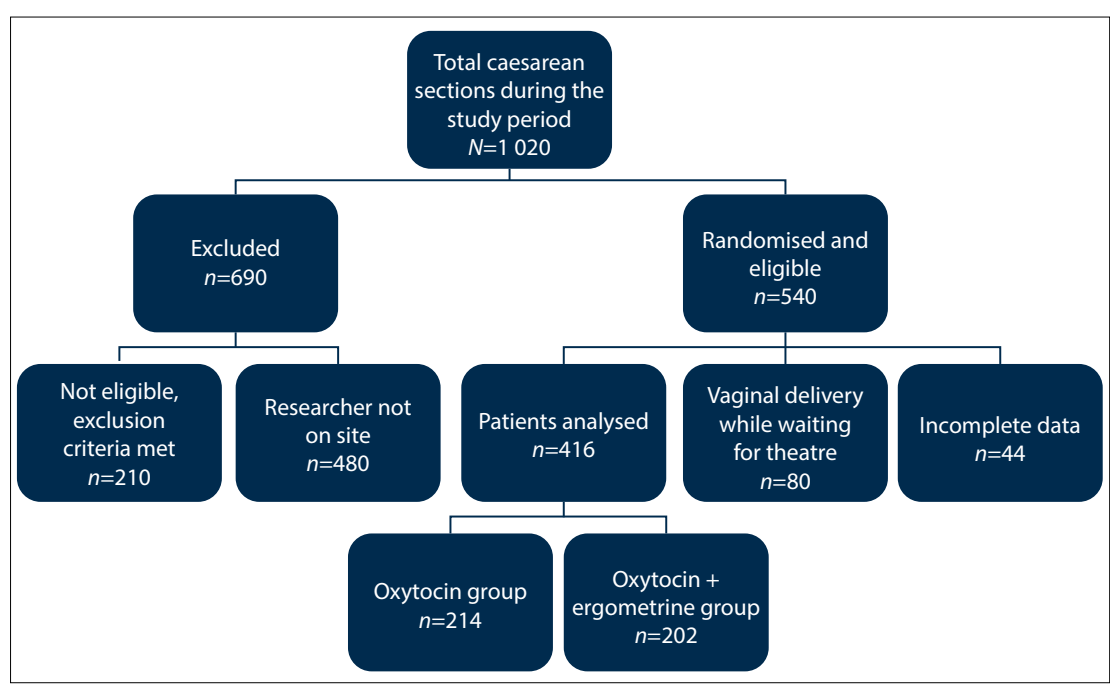

Fig. 1. Flow diagram of randomised patients.

Table 4. Comparison of emergency v. elective CSs

\begin{tabular}{llll}
\hline & $\begin{array}{l}\text { O group } \\
(\boldsymbol{N = 2 1 4 )}, \boldsymbol{n}(\mathbf{\%})\end{array}$ & $\begin{array}{l}\text { O+E group } \\
(\mathbf{N = 2 0 2}), \boldsymbol{n}(\%)\end{array}$ & $\begin{array}{l}\text { Total } \\
(\mathbf{N = 4 1 6 ) ,} \boldsymbol{n}(\%)\end{array}$ \\
\hline Emergency CS & $136(63.6)$ & $131(64.9)$ & $267(64.2)$ \\
Elective CS & $78(36.4)$ & $71(35.1)$ & $149(35.8)$ \\
O = oxytocin; O+E = oxytocin + ergometrine. & &
\end{tabular}

Table 5. Comparison of side-effects

\begin{tabular}{llll}
\hline Side-effect & $\begin{array}{l}\text { O group } \\
(\boldsymbol{n = 2 1 4}), \boldsymbol{n}(\%)\end{array}$ & $\begin{array}{l}\text { O+E group } \\
(\boldsymbol{n}=\mathbf{2 0 2}), \boldsymbol{n}(\%)\end{array}$ & $\boldsymbol{p}$-value \\
\hline Nausea & $23(11.7)$ & $26(12.9)$ & 0.722 \\
Vomiting & $5(2.3)$ & $11(5.4)$ & 0.092 \\
Headache & $20(9.3)$ & $20(9.9)$ & 0.065 \\
Palpitations & $9(4.2)$ & $17(8.4)$ & 0.294 \\
Dizziness & $29(13.6)$ & $28(13.9)$ & 0.867 \\
O = oxytocin; O+E = oxytocin + ergometrine. & & &
\end{tabular}

Table 6. Comparison of blood loss in the oxytocin v. oxytocin + ergometrine groups and need for blood transfusion

\begin{tabular}{llllll}
\hline & $\begin{array}{l}\text { Preoperative } \\
\text { haematocrit, } \\
\text { mean }(\mathrm{SD})\end{array}$ & $\begin{array}{l}\text { Postoperative } \\
\text { haematocrit, } \\
\text { mean }(\mathrm{SD})\end{array}$ & $\begin{array}{l}\text { Estimated visual } \\
\text { blood loss }(\mathbf{m L}), \\
\text { mean }(\mathrm{SD})\end{array}$ & $\begin{array}{l}\text { Calculated blood } \\
\text { loss }(\mathbf{m L}), \\
\text { mean }(\mathrm{SD})\end{array}$ & $\begin{array}{l}\text { Need for blood } \\
\text { transfusion, } \boldsymbol{n} \text { (\%) }\end{array}$ \\
\hline O group $(n=214)$ & $0.358(0.045)$ & $0.324(0.05)$ & $607(246)$ & $610(249)$ & $19(8.9)$ \\
O+E group $(n=202)$ & $0.359(0.045)$ & $0.329(0.045)$ & $588(236)$ & $590(245)$ & $7(3.5)$ \\
$\mathrm{O}=$ oxytocin; $\mathrm{O}+\mathrm{E}=$ oxytocin + ergometrine; $\mathrm{SD}=$ standard deviation. & & & &
\end{tabular}


Other data comparing oxytocin alone with the combination of ergometrine + oxytocin have shown a small but statistically significant reduction in the risk of $\mathrm{PPH}$ (odds ratio (OR) 0.82; $95 \%$ CI $0.71-0.95$ ), with no difference in the risk of severe PPH. Ergometrine + oxytocin was also associated with a reduced need for additional uterotonic drugs (OR 0.83; 95\% CI 0.72 - 0.96), with no difference in the risk of manual removal of the placenta. ${ }^{[7]}$ Four randomised trials compared different uterotonics for prophylaxis of PPH in women delivering by CS. In the UK, appraisal of these trials together with consideration of standard practice led to the development group for the National Institute for Health and Clinical Excellence (NICE) Caesarean Section Guideline. ${ }^{[10]}$

Results from this study showed no significant differences in terms of calculated or visual blood loss using oxytocin or oxytocin + ergometrine for prophylaxis of PPH at the time of CS. The oxytocin group had a non-significant trend towards a slightly higher calculated and visual blood loss. The most important finding of this study is that the need for blood transfusions (based on clinical indications) was significantly higher in the oxytocin group than in the oxytocin + ergometrine group $(p=0.01)$. Women in the oxytocin group were more than twice as likely to require a blood transfusion as those in the oxytocin + ergometrine group (RR 2.78; 95\% CI 1.21 6.4). A possible explanation for the similar estimated and calculated blood loss in the two groups is that the patients who received blood transfusions were excluded from both groups because their laboratory values were not sufficiently accurate for determination of calculated blood loss. The fact that there was no statistically significant difference between estimated and visual blood loss also suggests that the blood transfusions in the oxytocin-alone group were clinically indicated. There was no predefined haemoglobin or haematocrit concentration indicating blood transfusions, and the decision for transfusion was based on the treating obstetricians' and anaesthetists' clinical judgement in conjunction with haemoglobin concentrations and other appropriate clinical parameters, as is done in normal clinical practice.

There were no statistically significant differences in side-effects experienced between the two groups.

\section{Conclusions}

In an effort to reduce $\mathrm{PPH}$ at the time of CS, changing protocol from the use of oxytocin to oxytocin + ergometrine could influence overall maternal morbidity and mortality. The reduction in the need for blood transfusion by more than half in the oxytocin + ergometrine group is an important finding of this study.

\section{References}

1. Dyer R, van Dyk D, Dresner A. The use of uterotonic drugs during caesarean section. Int J Obstet Anesth 2010;19(3):313-319. DOI:10.1016/j.ijoa.2010.04.011

2. Cameron MJ, Robson SC. Vital statistics: An overview. In: Lynch CB, Keith LG, Lalonde AB, Karoshi M, eds. A Textbook of Postpartum Hemorrhage in Resource Poor Countries: A Comprehensive Guide to Evaluation, Management and Surgical Intervention. 1st ed. Kirkmahoe, Dumfriesshire, UK: Sapiens Publishing, 2006:17-34. http://www.sapienspublishing.com/pph_pdf/PPH.pdf (accessed 6 March 2016).

3. Pattinson RC, ed. Saving Mothers 2011-2013: The Sixth Report of the National Committee for Confidential Enquiries into Maternal Deaths in South Africa. Pretoria: Government Printer, 2014.

4. Royal College of Obstetricians and Gynecologists. Postpartum Haemorrhage: Prevention and Management (Green Top Guideline No. 52). https://www.rcog.org.uk/globalassets/documents/ guidelines/gt52postpartumhaemorrhage0411.pdf (accessed 6 March 2016).

5. Kovacheva VP, Soens MA, Tsen LC. A randomized, double-blinded trial of a 'rule of threes' algorithm versus continuous infusion of oxytocin during elective cesarean delivery. Anesthesiology 2015;123(1):92-100. DOI:10.1097/ALN.0000000000000682

6. Farina Z, Fawcus S. Management of obstetric haemorrhage: Oxytocin - ensuring appropriate use and balancing efficacy with safety. S Afr Med J 2015;105(4):271-274. DOI:10.7196/SAMJ.9179

7. National Committee for Confidential Enquiry into Maternal Deaths in South Africa. Saving Mothers 2008-2010: Fifth Comprehensive Report. Department of Health, 2012. http://www.hst.org.za/sites/ default/files/savingmothersshort.pdf (accessed 6 March 2016).

8. Stratford I, Dildy GA, Clark SL, Belfort MA. Visually estimated and calculated blood loss in vaginal and cesarean delivery. Am J Obstet Gynecol 2008;199(5):519.e1-7. DOI:10.1016/j.ajog.2008.04.049 9. Cooper G, Lewis G, Neilson J. Confidential enquiries into maternal deaths, 1997-1999. Br J Anaesth 2002;89(3):369-372. DOI:10.1093/bja/89.3.369

10. Wee MY, Brown H, Reynolds F. The National Institute of Clinical Excellence (NICE) guidelines for caesarean sections: Implications for the anaesthetist. Int J Obstet Anesth 2005;14(2):147-158. DOI:10.1016/j.jpoa.2004.09.008

Accepted 14 October 2015 